\title{
Ultrasonographic evaluation of the median nerve at the level of the carpal tunnel outlet and mid forearm in patients with type II Mucopolysaccharidosis
}

\author{
Corina Bocsa ${ }^{1}$, Carmen Asavoaie ${ }^{2}$, Simona Bucerzan ${ }^{3}$, Ioana Nascu ${ }^{3}$, Irina Brumboiu ${ }^{4}$, \\ Camelia Al-Khzouz ${ }^{3}$
}

1"Interservisan" Medical and Surgical Centre, ${ }^{2}$ Emergency Children Hospital, $1^{\text {st }}$ Pediatric Clinic, Department of Radiology and Imaging, ${ }^{3}$ Emergency Children Hospital, $1{ }^{\text {st }}$ Pediatric Clinic, Department of Genetics, "“iuliu Hatieganu" University of Medicine and Pharmacy, Epidemiology and Primary Health Care Cluj-Napoca, Romania

\begin{abstract}
Aims: The ultrasonographic (US) evaluation of the median nerve at the level of the carpal tunnel outlet (CTO) and mid forearm in pediatric patients with mucopolysaccharidosis type II (MPS II) and comparison with healthy subjects. Material and method: Fifteen children with MPS II and 44 healthy children were included in the study and they were divided into three age groups. The cross-sectional area, the appearance of the nerve, and the ratio of the cross-sectional areas were evaluated by US. Results: At the level of the CTO the mean area of the nerve was increased in all MPS II groups compared with the correspondent healthy age groups and the differences were statistically significant $(p<0.01)$. At the level of the mid forearm the differences were statistically significant only for the first age group. Other US findings at the level of the CTO in the MPS II groups were represented by hypoechogenicity $(86.67 \%$ on the right and $93.33 \%$ on the left), thickened fascicles $(80 \%$ bilaterally), irregular contour ( $53.33 \%$ bilaterally) and the presence of the Doppler signal including the nerve $(26.67 \%$ on the right and $33.33 \%$ on the left). The CTO/mid forearm cross-sectional area ratio was higher in all MPS II age groups and the differences were statistically significant $(\mathrm{p}<0.001)$. Conclusion: In patients with MPS II there are significant US changes in the size and aspect of the median nerve.
\end{abstract}

Keywords: mucopolysaccharidosis, median nerve, carpal tunnel outlet, mid forearm, ultrasonography

\section{Introduction}

Mucopolysaccharidoses (MPS) represent a group of rare inherited metabolic disorders caused by genetic defects that result in the absence or severe deficiency of one of the lysosomal hydrolases responsible for the degradation of glycosaminoglycans (GAGs) $[1,2]$. With the exception of MPS II (known as Hunter syndrome), which is an X-linked recessive disease, other lysosomal storage disorders are autosomal-recessive diseases [3]. MPS II occurs almost exclusively in males, but it has also been

Received 15.11.2015 Accepted 29.12.2015

Med Ultrason

2016, Vol. 18, No 1, 36-41

Corresponding author: Carmen Asavoaie

68 Motilor street

400370 Cluj-Napoca, Romania

E-mail: carmen.asavoaie@gmail.com reported in a small group of female patients. The most common mechanism for disease expression in female patients is thought to involve the process of X-chromosome inactivation [4].

MPS II is caused by a deficiency of iduronate2-sulfatase, which normally cleaves a sulfate group from GAGs, heparan and dermatan sulfate. A shortage of this enzyme leads to an accumulation of undegraded GAGs within the lysosomes of various tissues, resulting in dysfunction of multiple organs and systems, producing a broad spectrum of chronic and progressive clinical manifestations with significant variability in age of onset and rate of progression [3,5]. In patients with early progressive disease, central nervous system involvement, progressive airway disease, and cardiac disease are present. In those with slowly progressive disease, the central nervous system is not (or is minimally) affected. Additional findings in both forms of MPS II include: short 
stature, macrocephaly, macroglossia, hoarse voice, conductive and sensorineural hearing loss, hepato-splenomegaly, hernia, dysostosis multiplex, spinal stenosis, or carpal tunnel syndrome (CTS) [6].

Patients with Hunter syndrome often undergo surgical procedures at a younger age, before diagnosis, so Hunter syndrome should be suspected in young children with a history of surgical interventions, particularly for hernia or CTS [7].

The few studies that have been made reveal a high frequency of the CTS in patients with MPS [8,9]. The diagnosis is usually established based on the clinical exam or nerve conduction studies. However, in MPS the diagnosis may be delayed, as the presentation is commonly atypical. Many patients do not complain of numbness or pain, particularly early in the course of disease when verbal or intellectual limitations may pose additional challenges to the diagnosis [10].

Numerous recent papers have shown similar sensitivity and specificity of ultrasonography (US) and nerve conduction studies in the diagnosis of the CTS [11-17] but no study regarding the US examination of the median nerve in children with Hunter syndrome has been published.

The main objective of our study was to evaluate by US the median nerve in pediatric patients with MPS II and to compare the aspect with the findings of a healthy population. A secondary objective of the study consisted of the assessment of the median nerve US appearance at the level of the carpal tunnel outlet (CTO) and mid forearm in healthy pediatric patients of various age groups since there are no studies published to date regarding this issue according to the authors' knowledge.

\section{Material and method}

The study group consisted of 15 male patients with MPS II, aged between 3 years and 6 months old and 16 years and 9 months old. These are all the pediatric patients diagnosed with MPS II in Romania and they are in the evidence of the Genetics Department of the Emergency Children Hospital, Cluj-Napoca. The mean age of symptoms onset in our study group was 1.78 years $( \pm 0.79)$, the mean age at specific diagnosis was 4.68 years $( \pm 2.63)$ and the mean duration of enzyme replacement therapy was 2.26 years $( \pm 1.53)$. At the moment all patients included in the study are under enzyme replacement therapy. The excluding criteria were represented by other possible causes for CTS and previous surgery for CTS.

The control group consisted of 44 healthy boys, ages between 3 years and 5 months old and 17 years and 6 months old. These children were examined during the same period of time as the MPS II patients. The excluding criteria were: trauma or surgery involving the median nerve and other diseases that might involve or alter the appearance of the median nerve.

All the subjects, both MPS II and control group, were divided into three age groups according to the main developmental stages of childhood: pre-school age group - 3 to 7 years old (mean age $4.7 \pm 0.8$ and $5.08 \pm 1.22$, respectively), school age group - 7 to 13 years old (mean age $9.12 \pm 2.46$ and $10.56 \pm 2.12$, respectively), and puberty age group - 13 to 17 years old (mean age $15.13 \pm 1.25$ and $15.78 \pm 1.21$, respectively), each of the groups presenting specific developmental features. [18]. No statistically significant differences were present concerning the mean age between the groups (all $\mathrm{p}<0.05$ ).

All the examinations were performed on a Toshiba Xario V 2.0. US machine, using a linear probe, with frequencies ranging from 8 to $14 \mathrm{MHz}$. For the evaluation of the median nerve the US machine was preset at $14 \mathrm{MHz}$ when examining the CTO and at $8 \mathrm{MHz}$ when examining the median nerve at mid forearm. For vascularisation assessment we used Power Doppler mode interrogation (PRF 0.4-0.6 kHz).

The patients (from both groups) were placed with the forearm in a supine position and the wrist in a neutral position. The transducer was first placed in a transverse view at the level of the CTO, using the pisiform bone as a landmark, and then it was rotated $90^{\circ}$ in order to obtain the longitudinal view [19]. The cross-sectional area of the nerve was determined on the transverse image, using the trace area system of the US machine. The examiner traced the outline of the nerve inside the hyperechoic ring produced by the perineurium [20]. In the mid forearm the measurement was also performed on the transverse image in an identical manner. The patients were evaluated by two experimented sonographers, each performing three measurements at each of the four levels and providing the mean value as the final area. The ratio between the CTO and mid forearm areas was also determined.

The nerve was also analyzed qualitatively: echogenicity, aspect of the nerve fascicles, contour, and vascularization (present or absent), bilaterally in CTO and mid forearm, in both transverse and longitudinal view.

The examination of MPS patients and healthy children was performed after written informed consent of the parents or legal guardians was obtained and the study was conducted with the approval of the local Ethics Committee.

\section{Statistical analysis}

Databases for healthy children and MPS II patients were realized using Excel. The descriptive statistics was performed in Epi Info 7 and XLStat 2007. The results 
Ultrasonographic evaluation of the median nerve at the level of the carpal tunnel outlet...

were expressed as a mean \pm standard deviation (SD). Indicators for central tendency and the $t$ dispersion distribution were used to describe the patients and the t test was used for comparison with the healthy children. A value of $p<0.05$ was considered to be statistically significant.

The agreement between the two examiners which performed the US exams was assessed by using Cohen's Kappa coefficient. For the qualitative evaluation each parameter was considered separately for each of the 15 patients. The coefficient was determined only for the CTO since the measurements of the nerve at the mid forearm were identical.

\section{Results}

A total of 15 patients with MPS II and 44 healthy children of similar ages were included in the study.

The mean area of the median nerve at the level of the CTO was increased bilaterally in all MPS II patients groups as compared with the healthy groups (all $\mathrm{p}<0.01$ ). At the level of the mid forearm the differences between the MPS II patients and the healthy subjects was statistically significant only in the first group of age $(\mathrm{p}<0.01)$ (Table I).

The ratios between the CTO and mid forearm areas are presented in Table II.

The US qualitative assessment of the median nerve in MPS patients revealed the following alterations at the level of the CTO: hypoechogenicity $(86.67 \%$ on the right and $93.33 \%$ on the left, respectively), thickened fascicles (80\% bilaterally), irregular contour $(53.33 \%$ bilaterally), and the presence of Power Doppler signal (26.67\% on the right and $33.33 \%$ on the left, respectively). Similar findings were present at mid forearm, but the proportion of affected nerves was lower: hypoechogenicity in $6.7 \%$ of the nerves on both sides, thickened fascicles in $20 \%$ on the right and in $13.3 \%$ on the left, irregular contour $13.3 \%$ on both sides and no Power Doppler signal on either side. These US findings, in contrast with the normal aspect, are illustrated in figures 1 and 2 .

The analysis of the agreement between the two sonographers in determining the cross-sectional area revealed a very good interobserver agreement $(k=0.916)$.

\section{Discussions}

The CTS is the most frequent compressive peripheral neuropathy in adults [21,22], but in children it is a very rare entity, most often associated with lysosomal storage disease, and especially with MPS [23-25]. Studies that have already been published about the CTS in MPS II revealed a frequency of the syndrome as high as $96 \%$ [9].

At the moment there are no established guidelines for the diagnosis and treatment of CTS associated with MPS II. The diagnosis is usually made based on clinical signs

Table I. Comparison between median nerve cross-sectional areas of MPS II patients and healthy subjects groups measured at the level of the carpal tunnel outlet and mid forearm.

\begin{tabular}{|c|c|c|c|c|c|c|}
\hline \multirow[t]{2}{*}{ Indicator } & \multicolumn{2}{|l|}{ Group 1} & \multicolumn{2}{|l|}{ Group 2} & \multicolumn{2}{|l|}{ Group 3} \\
\hline & Healthy & MPS II & Healthy & MPS II & Healthy & MPS II \\
\hline Right CTO area $\left(\mathrm{mm}^{2}\right)$ & $4.5 \pm 0.97$ & $10.5 \pm 4.63$ & $5.56 \pm 0.63$ & $17.25 \pm 4.03$ & $9.42 \pm 1.78$ & $23.67 \pm 13.5$ \\
\hline $\mathrm{T}$ and $\mathrm{p}$ & \multicolumn{2}{|c|}{$5.075 ; \mathrm{p}<0.001$} & \multicolumn{2}{|c|}{$11.997 ; \mathrm{p}<0.001$} & \multicolumn{2}{|c|}{$3.981 ; \mathrm{p}<0.002$} \\
\hline Left CTO area $\left(\mathrm{mm}^{2}\right)$ & $4.62 \pm 1.02$ & $10.88 \pm 4.67$ & $5.44 \pm 0.81$ & $18 \pm 2.83$ & $8.75 \pm 1.91$ & $20 \pm 6.93$ \\
\hline $\mathrm{T}$ and $\mathrm{p}$ & \multicolumn{2}{|c|}{$5.221 ; \mathrm{p}<0.001$} & \multicolumn{2}{|c|}{$16.323 ; \mathrm{p}<0.001$} & \multicolumn{2}{|c|}{$5.383 ; \mathrm{p}<0.001$} \\
\hline Right $\mathrm{MF}$ area $\left(\mathrm{mm}^{2}\right)$ & $3.44 \pm 0.51$ & $4.88 \pm 1.55$ & $4.31 \pm 0.79$ & $5 \pm 0.82$ & $7 \pm 0.95$ & $9 \pm 3.61$ \\
\hline $\mathrm{T}$ and $\mathrm{p}$ & \multicolumn{2}{|c|}{$3.419 ; \mathrm{p}<0.01$} & \multicolumn{2}{|c|}{$1.548 ; \mathrm{p}>0.10$} & \multicolumn{2}{|c|}{$1.862 ; \mathrm{p}>0.05$} \\
\hline Left MF area $\left(\mathrm{mm}^{2}\right)$ & $3.69 \pm 0.79$ & $5.13 \pm 1.25$ & $4.63 \pm 0.89$ & $5.25 \pm 0.5$ & $6.58 \pm 1.31$ & $9.33 \pm 3.06$ \\
\hline $\mathrm{T}$ and $\mathrm{p}$ & \multicolumn{2}{|c|}{$3.461 ; \mathrm{p}<0.01$} & \multicolumn{2}{|c|}{$1.331 ; \mathrm{p}>0.10$} & \multicolumn{2}{|c|}{$2.506 ; \mathrm{p}<0.05$} \\
\hline
\end{tabular}

The results are expressed as mean area \pm SD. Group 1 - 3 to 7 years old; Group 2 - 7 to 13 years old ; Group 3 - 13 to 17 years old ; CTO carpal tunnel outlet; $\mathrm{MF}$ - mid forearm;

Table II. Comparison of the carpal tunnel outlet /mid forearm ratios between healthy subjects and MPS II patients.

\begin{tabular}{|c|c|c|c|c|c|c|}
\hline \multirow[t]{2}{*}{ Indicator } & \multicolumn{2}{|l|}{ Group 1} & \multicolumn{2}{|l|}{ Group 2} & \multicolumn{2}{|l|}{ Group 3} \\
\hline & Healthy & MPS II & Healthy & MPS II & Healthy & MPS II \\
\hline Right $\mathrm{CTO} / \mathrm{MF}$ areas ratio & $1.30 \pm 0.15$ & $2.32 \pm 1.29$ & $1.32 \pm 0.23$ & $3.45 \pm 0.64$ & $1.35 \pm 0.18$ & $2.49 \pm 0.55$ \\
\hline $\mathrm{T}$ and $\mathrm{p}$ & \multicolumn{2}{|c|}{$3.208 ; \mathrm{p}<0.02$} & \multicolumn{2}{|c|}{$11.426 ; \mathrm{p}<0.001$} & \multicolumn{2}{|c|}{$6.556 ; \mathrm{p}<0.001$} \\
\hline Left $\mathrm{CTO} / \mathrm{MF}$ areas ratio & $1.27 \pm 0.25$ & $2.13 \pm 0.83$ & $1.21 \pm 0.27$ & $3.45 \pm 0.64$ & $1.34 \pm 0.23$ & $2.13 \pm 0.23$ \\
\hline $\mathrm{T}$ and $\mathrm{p}$ & \multicolumn{2}{|c|}{$3.865: p<0.001$} & \multicolumn{2}{|c|}{$11.220 ; \mathrm{p}<0.001$} & \multicolumn{2}{|c|}{$5.416 ; \mathrm{p}<0.001$} \\
\hline
\end{tabular}

The results are expressed as mean are \pm SD. Group 1 - 3 to 7 years old; Group 2 - 7 to 13 years old ; Group 3 - 13 to 17 years old ; CTO carpal tunnel outlet; MF - mid forearm; SD - standard error 


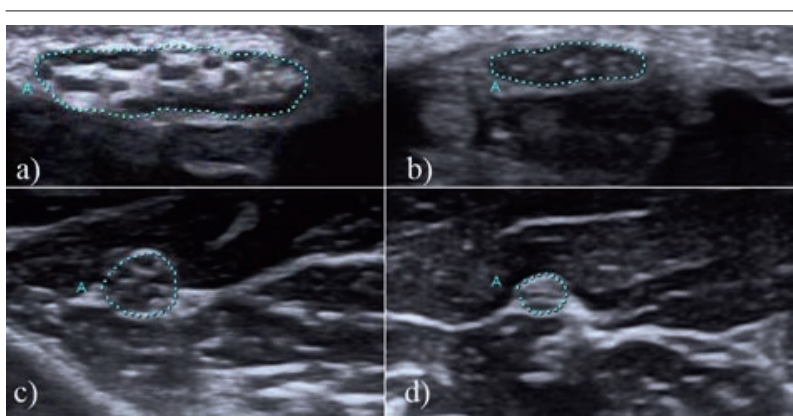

Fig 1. US of the median nerve at the level of the CTO, pathological appearance and cross-sectional area in a patient with MPS II, cross sectional area $37 \mathrm{~mm}^{2}$ (a), in contrast with the normal aspect of the nerve in a healthy boy of a similar age, cross sectional area $7 \mathrm{~mm}^{2}$ (b). US transversal scan at the level of the mid forearm, pathological aspect in a patient with MPS II (c) and a normal healthy boy of the same age (d). The cross sectional area is increased in the MPS patient and the fascicles are thicker and hypoechoic.

in association with nerve conduction studies. While the clinical signs described by the adults with CTS are well known and frequent, they can be very subtle or missing in children with MPS: decreased sweating, night time waking, gnawing of the hands, or manual clumsiness [26]. Also, these symptoms may be overlooked due to other specific alterations seen in MPS II, such as skeletal dysplasia or articular pain. Also some of the children are either too small or present significant neurological impairment, which prevents them from accurately communicating their symptoms.

The electrophysiological examination is very useful in the diagnosis and evaluation of the severity of the CTS, but may be difficult to be performed in children with MPS II and it must be adapted to the clinical characteristics of the patients [26].

More and more studies have proven that US is an accurate, low cost imaging method that can be used in the evaluation of the CTO anatomy and that is more easily accepted by parents and patients. The US findings associated with the CTS have been described in adults and they consist of the increased size of the median nerve proximal to the flexors retinaculum, sudden change in size at the level of the tunnel outlet, decreased echogenicity, decreased mobility and increase of the Doppler signal $[27,28]$. According to the knowledge of the authors no studies regarding the US evaluation of the median nerve in MPS II patients have been published. There is a case report of an adult MPS II patient with end-stage CTS published by Alkhachroum et al which reveals the same US findings and acknowledges the role of US in identifying the median nerve and its alterations associated with CTS in MPS II patients. [29]

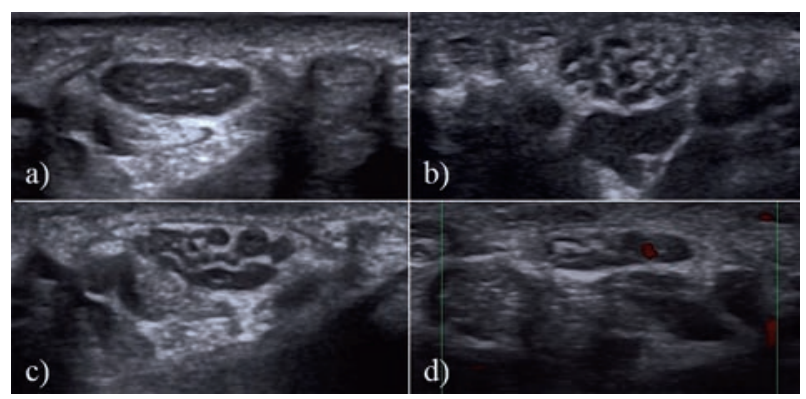

Fig 2. US transverse scan of the median nerve at the level of the CTO. All images reveal increased cross-sectional area of the nerve, image a) present the hypoechoic aspect of the nerve, b) and c) show the markedly thickened, individualized fascicles of the nerve, in c) the nerve has an irregular contour, while in d) it is flattened and has Power Doppler signal insight of the nerve.

A meta-analysis found that the sensitivity and specificity of US in establishing the CTS diagnosis in adults, is $77.6 \%$ and $86.8 \%$, respectively [30].

The results of our study correlate with literature regarding the type of changes found in CTS in adults. Obviously, due to the rather small number of patients examined, further studies are necessary, especially multicentric studies, due to the small number of patients that can be found in a single center.

The values of the cross-sectional area of the median nerve proximal to the carpal tunnel outlet that are considered pathological are different depending on the examiner and the inclusion or not of the epineurium in the measurement. As Klauser et al demonstrated, the measurement of the median nerve cross-sectional area in two different sites and the calculation of the differences between the two sites increases the accuracy of the CTA diagnosis [31]. According to this idea it has been suggested that the ratio between the cross-sectional area of the median nerve at the level of the CTO and the forearm may be a more precise indicator in establishing the diagnosis $[32,33]$. Hobson-Webb et al found that the cross-sectional area ratio in patients with CTS is higher $(2.1 \pm 0.5)$ than in asymptomatic patients $(1.0 \pm 0.1)$, a 1.4 ratio having a $100 \%$ sensitivity for the detection of CTS patients, while using just the cross-sectional area of the nerve at the level of the carpal tunnel outlet has $45-93 \%$ sensitivity, depending on the reference value which was used [32]. We found a significantly higher ratio in healthy subjects than in MPS II patients, while the variation coefficient and the lower standard deviation compared with the wrist measurements may suggest that the $\mathrm{CTO}-$ mid forearm ratio is superior in assessing the alterations of the nerve size in MPS II.

Intraneural vascularity has been proposed as an additional parameter in the diagnosis of CTS, sometimes 
being the only detectable finding $[34,35]$. Recent studies have shown that endoneural hypervascularity is correlating with the severity of the median nerve lesions evaluated through electrophysiologic studies [36,37]. Dalmau Serra et al demonstrated the recovery from CTS of a MPS II patient after enzyme replacement therapy was introduced [38]. In our study group the Doppler signal was present in only a few patients and this finding could be explained by the fact that almost all of them were under enzyme replacement therapy for various lengths of time. Of course this hypothesis requires further studies also.

Regarding the development of the CTS in MPS II patients there have been only a few hypotheses formulated: the compression of the nerve caused by the thickening of the flexors retinaculum and of the tissue surrounding the tendons sheath due to abundant accumulation of GAGs, or the significant alteration of the local anatomy caused by the bone dysplasia $[23,39,40]$. The presence of statistically significant changes in the median nerve at the level of the forearm may suggest that the structural changes are, also, caused by the disease itself, and it is not just the compression inducing the changes at the level of the carpal tunnel outlet. Since the histopathological exam is difficult to perform due to ethical issues, further studies will be necessary to confirm these hypotheses.

Our opinion is that in MPS II patients the median nerve area determined by US could be useful for monitoring the response to the treatment (enzyme replacement therapy). In order to do this objectively a US scoring system that will include quantifiable findings (cross-sectional area, hypoechogenicity, thickened fascicles, irregular contour, and presence of Power Doppler signal) needs to be defined in the future.

Our study has some limitations. Since MPS II is a rare disease, the number of patients included in our study is obviously small and that lead to a reduced number of median nerves available for evaluation. Another inconvenience was represented by the fact that the patients had been diagnosed and treated for various periods of time and they present different forms of the disease, leading to a rather heterogeneous group. Nevertheless, we believe that the significant median nerve alterations found in the majority of the MPS II patients in our group overcome these limitations and further studies, maybe multicentric, will be able to establish more accurately the role of US in the diagnosis and monitoring of the MPS II patients.

\section{Conclusions}

US is an imaging method that may be considered as a first line investigation in the assessment of the median nerve in MPS II patients as it produces highly accurate results in detecting nerve alterations (increased size, hypoechogenicity, thickened fascicles, irregular contour and presence of Power Doppler signal) associated with MPS II. The CTO/mid forearm cross sectional areas ratio is significantly higher in patients with MPS II than in normal subjects and it seems it is superior in the appreciation of the size of the median nerve in patients with MPS II, promising to be a valid method of the CTS diagnosis.

\section{Conflict of interest: none}

\section{References}

1. Cimaz R, La Torre F. Mucopolysaccharidoses. Curr Rheumatol Rep 2014; 16: 389.

2. Muenzer J. Overview of the mucopolysaccharidoses. Rheumatology (Oxford) 2011; 50 Suppl 5: v4-v12.

3. Neufeld EF, Muenzer J. The mucopolysaccharidoses. In: Scriver CR, Beandet AL, Sly WS, Valle D, editors. The Metabolic and Molecular Bases of Inhereted Disease. 8th edition. III. Mc Graw-Hill; New York 2001: 3421-3452.

4. Jurecka A, Krumina Z, Żuber Z, et al. Mucopolysaccharidosis type II in females and response to enzyme replacement therapy. Am J Med Genet A 2012; 158A: 450-454.

5. Giugliani R, Villarreal ML, Valdez CA, et al. Guidelines for diagnosis and treatment of Hunter Syndrome for clinicians in Latin America. Genet Mol Biol 2014; 37: 315-329.

6. Scarpa M, Almássy Z, Beck M, et al; Hunter Syndrome Europena Expert Council. Mucopolysaccharidosis type II: European recommendations for the diagnosis and multidisciplinary management of a rare disease. Orphanet J Rare Dis 2011; 6: 72 .

7. Mendelsohn NJ, Harmatz P, Bodamer O, et al; Hunter Outcome Survey Investigators. Importance of surgical history in diagnosing mucopolysaccharidosis type II (Hunter syndrome): data from the Hunter Outcome Survey. Genet Med 2010; 12: 816-822.

8. Sharma R, Ramachandran S, Bhat D, Bindu PS, Devi BI. Bilateral carpal tunnel syndrome in two children: common manifestation of an uncommon disease. Pediatr Neurosurg 2013; 49: 300-302.

9. Kwon JY, Ko K, Sohn YB, et al. High prevalence of carpal tunnel syndrome in children with mucopolysaccharidosis type II (Hunter syndrome). Am J Med Genet A 2011; 155A: 1329-1335.

10. Yuen A, Dowling G, Johnstone B, Kornberg A, Coombs C. Carpal tunnel syndrome in children with mucopolysaccaridoses. J Child Neurol 2007; 22: 260-263.

11. Azami A, Maleki N, Anari H, Iranparvar Alamdari M, Kalantarhormozi M, Tavosi Z. The diagnostic value of ultrasound compared with nerve conduction velocity in carpal tunnel syndrome. Int J Rheum Dis 2014; 17: 612-620.

12. McDonagh C, Alexander M, Kane D. The role of ultrasound in the diagnosis and management of carpal tunnel syndrome: a new paradigm. Rheumatology (Oxford) 2015; 54: 9-19. 
13. Fowler JR, Munsch M, Tosti R, Hagberg WC, Imbriglia JE. Comparison of ultrasound and electrodiagnostic testing for diagnosis of carpal tunnel syndrome: study using a validated clinical tool as the reference standard. J Bone Joint Surg Am 2014; 96: e148.

14. Fowler JR, Cipolli W, Hanson T. A Comparison of Three Diagnostic Tests for Carpal Tunnel Syndrome Using Latent Class Analysis. J Bone Joint Surg Am 2015; 97: 19581961.

15. Fowler JR, Gaughan JP, Ilyas AM. The sensitivity and specificity of ultrasound for the diagnosis of carpal tunnel syndrome: a meta-analysis. Clin Orthop Relat Res 2011; 469: 1089-1094.

16. Pastare D, Therimadasamy AK, Lee E, Wilder-Smith EP. Sonography versus nerve conduction studies in patients referred with a clinical diagnosis of carpal tunnel syndrome. J Clin Ultrasound 2009; 37: 389-393.

17. Visser LH, Smidt MH, Lee ML. High-resolution sonography versus EMG in the diagnosis of carpal tunnel syndrome. J Neurol Neurosurg Psychiatry 2008; 79: 63-67.

18. Kail R V, Children and their Development. 6th edition. (Mydevelopmentlab Series). Engelwood Cliffs, N.J: Prentice Hall, 2011.

19. Martinoli C, Bianchi S, Cohen M, Graif M. Ultrasound of peripheral nerves. J Radiol 2005; 86: 1869-1878.

20. Alves MPT, Fonseca COP, Grajeiro JM. Carpal tunnel syndrome: comparative study between sonographic and surgical measurements of the median nerve in moderate and severe cases of disease. Radiol Bras 2013; 46: 23-29.

21. Alfonso C, Jann S, Massa R, Torreggiani A. Diagnosis, treatment and follow-up of the carpal tunnel syndrome: a review. Neurol Sci 2010; 31: 243-252.

22. Atroshi I, Englund M, Turkiewicz A, Tägil M, Petersson IF. Incidence of physician-diagnosed carpal tunnel syndrome in the general population. Arch Intern Med 2011; 171: 943 944.

23. Van Meir N, De Smet L. Carpal tunnel syndrome in children. Acta Orthop Belg 2003; 69: 387-395.

24. Héron B. Neurologic manifestations in mucopolysaccharidoses. Arch Pediatr 2014; 21 Suppl 1: S14-S21.

25. Bona I, Vial C, Brunet P, et al. Carpal tunnel syndrome in Mucopolysaccharidoses. A report of four cases in child. Electromyogr Clin Neurophysiol 1994; 34: 471-475.

26. White K, Kim T, Neufeld JA. Clinical assessment and treatment of carpal tunnel syndrome in the mucopolysaccharidoses. J Pediatr Rehabil Med 2010; 3: 57-62.

27. Lawande AD, Warror SS, Jashi MS. Role of ultrasound in evaluation of peripheral nerves. Indian J Radiol Imaging 2014; $24: 254-258$.
28. Ghasemi-Rad M, Nosair E, Vegh A, et al. A handy review of carpal tunnel syndrome: From anatomy to diagnostic and tratament. World J Radiol 2014; 6: 284-300.

29. Alkhachroum AM, Preston DC. Ultrasound findings of carpal tunnel syndrome in a hunter syndrome patient. Muscle Nerve 2016; 53: 147-150.

30. Fowler JR, Gaughan JP, Ilyas AM. The sensitivity and speciticity of ultrasound for the diagnosis of carpal tunnel syndrome: a meta-analysis. Clin Orthop Relat Res 2011; 469: 1089-1094.

31. Klauser AS, Halpern EJ, De Zordo T, et al. Carpal tunnel syndrome assessment with US: value of additional crosssectional area measurements of the median nerve in patients versus healthy volunteers. Radiology 2009; 250: 171-177.

32. Hobson-Webb LD, Massey JM, Juel VC, Sanders DB. The ultrasonographic wrist-to-forearm median nerve area ratio in carpal tunnel syndrome. Clin Neurophysiol 2008; 119: 1353-1357.

33. Abrishamchi F, Zaki B, Basiri K, Ghasemi M, Mohaghegh $\mathrm{M}$. A comparison of the ultrasonographic median nerve crosssectional area at the wrist and the wrist-to-forearm ratio in carpal tunnel syndrome. J Res Med Sci 2014; 19: 1113-1117.

34. Mallouhi A, Pulzl P, Trieb T, Piza H, Bodner G. Predictors of carpal tunnel syndrome: accuracy of gray-scale and color Doppler sonography. AJR AM J Roentgenol 2006; 186: $1240-1245$.

35. Joy V, Therimadasamy AK, Chan YC, Wilder-Smith EP. Combined Doppler and B-mode sonography in carpal tunnel syndrome. J Neurol Sci 2011; 308: 16-20.

36. Mohammadi A, Ghasemi-Rad M, Mladkova-Suchy N, Ansari S. Correlation between the severity of carpal tunnel syndrome and color Doppler sonography findings. AJR Am J Roentgenol 2012; 198: W181-W184.

37. Evans KD, Roll SC, Volz KR, Freimer M. Relationship between intraneural vascular flow measured with sonography and carpal tunnel syndrome diagnosis based on electrodiagnostic testing. J Ultrasound Med 2012; 31: 729-736.

38. Dalmau Serra J, Vitoria Miñana I, Calderón Fernández R, Cortell Aznar I. Clinical response to long term enzyme replacement treatment in children, adolescent and adult patients with Hunter syndrome. Med Clin (Barc) 2015; 145: 392-398.

39. Meyer-Marcotty MV, Kollewe K, Dengler R, Grigull L, Altintas MA, Vogt PM. Carpal tunnel syndrome in children with mucopolysaccharidosis type $1 \mathrm{H}$ : diagnosis and therapy in an interdisciplinary centre. Handchir Mikrochir Plast Chir 2012; 44: 23-28.

40. Morishita K, Petty RE. Musculoskeletal manifestations of mucopolysaccaridoses. Rheumatology 2011; 50: v19-v25. 